\title{
Refractive index sensor based on etched D-Fibres with wavelength adjustable operating point and resolution
}

\author{
Sameer M. Chandani ${ }^{*}$, Nicolas A.F. Jaeger \\ Dept. of Electrical \& Computer Engineering, University of British Columbia, Vancouver, BC, \\ Canada
}

\begin{abstract}
Silica based fibre-optic refractive index sensors are gaining acceptance over conventional refractometers and finding applications in chemical/biological sensing due to many of their desirable properties. Here we present an optical fibrebased refractive index sensor that uses the power transmission through etched D-shaped fibres. The sensor's operating point and resolution can be tailored for a specific application by selecting the correct combination of the operating wavelength and the cladding thickness of the etched fibre. The sensor's power transmission depends on the surrounding refractive index in which the sensor head is immersed. The sensor presented has a maximum resolution on the order of $10^{-6}$ in its "high resolution" region and on the order of $10^{-4}$ in its "low resolution" region. The refractive index at which the maximum resolution occurs in the high resolution region can be shifted by $\sim 0.012$ and by $\sim 0.027$ in the low resolution region. To date, such high resolutions have been reported over narrow ranges and/or for fibre Bragg grating based sensors, which require optical spectral analysis which typically, is costly.
\end{abstract}

Keywords: D-fibre, refractive index sensor, optical fibre sensors

\section{INTRODUCTION}

Optical fibre-based refractive index sensors are promising and attractive for use in chemical and biological sensing applications due to their inherent advantages such as chemical inertness, high sensitivity, compactness, light weight, non-intrusiveness, immunity to electromagnetic fields, and fast response times. The measurement of the refractive index of many chemical substances can be used to determine certain physical parameters, such as density, $\mathrm{pH}$, acidity, and liquid concentration ${ }^{1,2}$. For these reasons, there has been a significant amount of work done toward the development of optical fibre-based refractive index sensors ${ }^{3,2-27}$.

There are two common methods of measurement used in optical fibre-based refractive index sensors. The first method uses wavelength interrogation and requires the measurement of the transmission spectrum of the sensor with the use of a broadband source and an optical spectrum analyzer. The resolution of such sensors is determined by the resolution to which shifts in the resonance wavelengths of the sensors can be measured. Such sensors have been demonstrated in a uniformly thinned fibre Bragg grating (FBG) obtained by using wet chemical etching ${ }^{4}$, in a thinned section of fibre (approximate radius of $\sim 1.5 \mu \mathrm{m}$ ) sandwiched between two FBGs used to create a fibre Fabry-Pérot interferometer ${ }^{5}$, in the resonance of cladding modes in a thinned $\mathrm{FBG}^{6}$, in tilted $\mathrm{FBGs}^{7,8}$, in $\mathrm{FBGs}$ written on side polished fibres ${ }^{9}$, on etched D-fibres, ${ }^{3,10}$ and on microstructured fibres ${ }^{11}$ and in long-period fibre gratings ${ }^{12,13,14}$ written on circular fibres. In Table 1 we have tabulated the peak sensitivities and operating points of the sensors described above for which have been reported. The range of refractive index values that can be measured for the majority of such sensors reported thus far is typically from 1.33 to 1.46 .

The second method uses intensity measurements and requires the measurement of the power transmission through the sensor using typical optical sources and detectors. In this method, the resolution depends on the resolution to which the optical power transmission can be measured. Such sensors have been demonstrated in a bent plastic multimode optical fibre with a partially stripped cladding ${ }^{15}$, in cladded multimode tapered fibre tips with mirrored ends based on the radiation of the modes guided by the cladding ${ }^{16}$, in an evanescent wave-based sensor created using a tapered single-mode fibre embedded in a polymer ${ }^{17}$, in the leakage of a side-polished single-mode fibre ${ }^{18}$, in side-polished plastic optical fibres ${ }^{19,20}$, in a microstructured fibre Bragg grating in a single-mode fibre ${ }^{21}$ (the wavelength of the optical source had to

\footnotetext{
*sameerc@ece.ubc.ca; phone 1-604-822-6268; fax 1-604-822-5949
}

Photonics North 2008, Réal Vallée, Michel Piché, Peter Mascher, Pavel Cheben, Daniel Côté, Sophie LaRochelle, Henry P. Schriemer, Jacques Albert, Tsuneyuki Ozaki, Eds., Proc. of SPIE Vol. 7099, 709924, (2008) - 0277-786X/08/\$18 - doi: 10.1117/12.806231 
be tuned to be close to the Bragg wavelength), in the fibre core diameter mismatches when a small section of a singlemode fibre was sandwiched between two lead multimode fibres ${ }^{2}$, in $\mathrm{V}$-shaped unclad multimode fibres with varying angles ${ }^{22}$, in a tapered section of multimode fibre sandwiched between two single-mode fibres having two different $\mathrm{FBGs}^{23}$. The ranges of operation and the peak resolution reported for the sensors described above are given in Table 2. Another specialized group of refractive index sensors includes surface plasmon resonance sensors ${ }^{24}$ which use wavelength interrogation ${ }^{25}$, intensity measurement ${ }^{26}$, or the phase difference between two orthogonal modes ${ }^{27}$.

Table 1: Reported peak sensitivities and operating points for refractive index sensors based on wavelength interrogation taken from the literature.

\begin{tabular}{ccc}
\hline \hline Peak Sensitivity $(\mathrm{nm} / \mathrm{RIU})$ & Operating Point (RIU) & Reference \\
\hline$\sim 600$ & $\sim 1.45$ & 3 \\
340 & 1.333 & 7 \\
300 & 1.455 & 13 \\
294 & 1.42 & 14 \\
$\sim 185$ & $\sim 1.35$ & 6 \\
172 & $\sim 1.45$ & 11 \\
143 & $\sim 1.45$ & 5 \\
71.2 & 1.333 & 4 \\
60 & $\sim 1.450$ & 8 \\
39 & 1.43 & 8 \\
\hline \hline
\end{tabular}

Table 2: Reported ranges or operating points and peak resolutions for refractive index sensors based on intensity variations taken from the literature.

\begin{tabular}{ccc}
\hline \hline Peak Resolution (RIU) & Range/Operating Point (RIU) & Reference \\
\hline $10^{-5}$ & $1.3333-1.4206$ & 23 \\
$3 \times 10^{-5}$ & $1.36-1.46$ & 16 \\
$7 \times 10^{-5}$ & $1.445-1.455$ & 21 \\
$4 \times 10^{-5}$ and $6 \times 10^{-5}$ & 1.41 and 1.38 & 22 \\
$2.5 \times 10^{-4}$ & $1.35-1.46$ & 18 \\
$10^{-4}$ & $1.46-1.48$ & 17 \\
$\sim 5 \times 10^{-4}$ & $1.37-1.40$ & 15 \\
$\sim 10^{-3}$ & $1.440-1.446$ & 19 \\
$10^{-3}$ & $>1.417$ & 20 \\
$2 \times 10^{-3}$ & $1.45-1.5$ & 20 \\
\hline \hline
\end{tabular}

In this paper we present a high resolution, wide range, intensity-based refractive index sensor that can be tailored with regard to its operating point and resolution. In Section 2 we describe the operating principle and a study of the effects of the cladding thickness and the operating wavelength on the resolution and operating point of the sensor. In Section 3 an experimental analysis of the sensor is presented, demonstrating the ability to fabricate a sensor with a particular operating point and resolution by carefully selecting the etched cladding thickness and the operating wavelength. A discussion is provided in Section 4 with concluding remarks given in Section 5.

\section{OPERATING PRINCIPLES}

Fig. 1 shows a conceptual diagram of the proposed sensor which is a D-fibre with a section of length $L$, having a cladding thickness $d$, which is defined as the minimum distance between the fibre core edge and the fibre flat. The etched section constitutes the sensor head.

The D-fibres used here (obtained from KVH Industries) were polarization maintaining and had elliptical cores. Before etching, our fibres had a cladding thickness of $d \approx 13 \mu \mathrm{m}$ and an elliptical core of semimajor axis $a \approx 2 \mu \mathrm{m}$ and semiminor axis $b \approx 1 \mu \mathrm{m}$. The core of the fibre was germania-doped with a refractive index $n_{c o}=1.4756$ at $\lambda_{o}=1550 \mathrm{~nm}$ and the cladding was fluorine-doped with a refractive index $n_{c l}=1.4410$ at $\lambda_{\mathrm{o}}=1550 \mathrm{~nm}$.

In a typical optical fibre, the fibre mode effective index $n_{\text {eff }}$ lies between the cladding refractive index $n_{c l}$ and the core refractive index $n_{c o}$. In the case of our D-fibre with a surrounding refractive index, $n_{s}, n_{\text {eff }}$ is changed, and for $n_{s} \geq n_{\text {eff }}$, the mode becomes leaky ${ }^{28,29,30,31,32}$. For the case where $n_{s}>n_{\text {eff }}$, the propagation constant for the structure's leaky $\mathrm{TE}_{0}$ 
mode is complex, with real and imaginary parts, $\beta_{r}$ and $\beta_{j}$, respectively. Consequently, the transmission through the sensor head depends on $n_{s}$ and thus an intensity-based refractive index sensor can be realized. The D-fibre/SRI structure can be theoretically modeled by first replacing the fibre with a three-layer equivalent planar waveguide by matching modal fields and propagation constants ${ }^{33}, 34$. This equivalent planar waveguide is then extended to a four-layer planar waveguide to take into account the SRI, which acts as a superstrate. The power transmission, $T_{P}$, can be written in terms of the power into $P_{i n}$, and the power out of $P_{\text {out }}$, a section of fibre of length $L$, as

$$
T_{P}=\frac{P_{o u t}}{P_{\text {in }}}=e^{-2 \beta_{j} L}
$$

Equation (1) has been used to calculate the power transmission ratio, $T_{P}$, of the equivalent planar waveguide's $\mathrm{TE}_{0}$ mode for a $1 \mathrm{~cm}$ length of the D-fibre sensor as a function of $n_{s}$ for several values of $d$ at $\lambda_{\mathrm{o}}=1550 \mathrm{~nm}$, as shown in Fig. 2. The sensor has three regions of operation, separated by the vertical dotted lines in Fig. 2 . The sensor has three regions of operation, separated by the vertical dotted lines in Fig. 2 . In Region I, where $n_{s} \gtrsim n_{\text {eff, the transmission decreases }}$ sharply to a minimum and the slope is large and negative over a small refractive index range. In Region II, where $n_{s}>n_{\text {eff }}$, the transmission is close to its minimum and its slope is small or even zero. In Region III, where $n_{s}>n_{\text {eff }}$ as well, the transmission increases monotonically and the slope is positive over a large refractive index range.

The resolution to which the refractive index can be measured, $\Delta n_{s}$, depends on the resolution to which $T_{P}$ can be measured, $\Delta T_{P}$, and is approximated by

$$
\Delta n_{s} \approx \Delta T_{P}\left(\frac{\partial T_{P}}{\partial n_{s}}\right)^{-1}
$$

It is apparent from (2) that the sensor resolution improves with increasing slope of $T_{P}$. The sensor is most useful in Regions I and III and the maximum resolutions in these two regions, calculated using (2), are shown in Fig. 3 for four values of $\Delta T_{P}$. In Region I the maximum resolution dramatically improves with decreasing $d$ and occurs at $n_{s} \approx 1.4495$ for all values of $d$. In Region III, however, the maximum resolution has a parabolic dependence on $d$ with its best value at the apex of the parabola which occurs at $d \approx 4.0 \mu \mathrm{m}$ indicating that there is an optimum cladding thickness, $d_{o p t}$, for such D-fibre sensors that gives the best resolution. The SRI at which the maximum resolution occurs, $n_{\max }$, is different for each $d$ and is marked by the circles in Fig. 2. The calculated maximum resolution in Region I is on the order of $10^{-6}$ and such resolutions have, thus far only been reported for fibre Bragg grating based sensors ${ }^{7,14}$ and surface plasmon resonance sensors ${ }^{25,27}$. Fig. 4 shows the calculated resolutions in Regions I and III for a sensor with $d=4.0 \mu \mathrm{m}$ as a function of $n_{s}$ for several values of $\Delta T_{P}$. The calculated resolution in Region III is on the order of $10^{-2}$ for $\Delta T_{P}=1 \%$ over a very large range. The figure shows that as expected, by improving $\Delta T_{P}$ by an order of magnitude, the resolution is also improved by an order of magnitude. In our case, we are able to achieve $\Delta T_{P} \leq 0.2 \%$, with no extra effort, which results in a resolution between $10^{-3}$ and $10^{-4}$ over a large range of 0.23 refractive index units (RIUs). For refractive indices other than $n_{\max }$ in Region III, the resolution is lower and the optimum cladding thickness is changed, thus, depending on the sensor's operating point its resolution can be maximized by selecting the optimal cladding thickness.

To better exploit the high resolutions obtainable in Region I, it is possible to shift the operating point by changing the operating wavelength $\lambda_{0}$. To demonstrate the effect on the sensor of changing the wavelength, Fig. 5 shows the calculated power transmission ratio of the equivalent planar waveguide's $\mathrm{TE}_{0}$ mode in Region $\mathrm{I}$ for a $1 \mathrm{~cm}$ length of our D-fibre sensor as a function of $n_{s}$ at several wavelengths using $d=4.0 \mu \mathrm{m}$. For each wavelength, the refractive index distribution of the fibre was determined using the equations for germania-doped and fluorine-doped silica ${ }^{35}$. The range over which Region I can be shifted is $\sim 0.012$ RIUs, which is sufficiently large for applications that require the accurate measurement of the refractive indices of the many liquids, such as typical hydrocarbons, for which $1.45 \leq n_{s} \leq 1.46$.

The maximum resolution point in Region III, i.e., $n_{\max }$, can also be shifted by changing $\lambda_{\mathrm{o}}$. Here, $d_{\text {opt }}$, is a function of both the operating point and the wavelength. Fig. 6 shows $d_{o p t}$ as a function of $n_{s}$ for several wavelengths. The $d_{\text {opt }}$ for each $\lambda_{0}$ is significantly different and shown in Fig. 7 are the power transmission curves at several wavelengths using the $d_{\text {opt }}$ for $\lambda_{\mathrm{o}}$. The figure's legend shows $d_{\text {opt }}$ and the corresponding $n_{\max }$ for each $\lambda_{0}$. Here, $n_{\max }$ can be shifted by $\sim 0.027$ RIUs, which is a significant amount considering the number of liquids that have refractive indices between 1.46 and 1.50, such as glycerin, benzene, chloroform, honey, turpentine, and sugar solutions. Fig. 2 to Fig. 7 illustrate that, in 
principle, a sensor having a desired resolution and operating point can be realized by carefully selecting the correct combination of the cladding thickness and the operating wavelength.

Furthermore, a tuneable broadband sensor can also be realized by picking a particular cladding thickness. Fig. 8 shows $T_{P}$ curves in Region I for $d=2.50 \mu \mathrm{m}$ at several wavelengths illustrating that $\lambda_{\mathrm{o}}$ can be tuned to change the sensor's operating point over the range $1.4462 \leq n_{s} \leq 1.4583$. For $\Delta T_{P}=0.2 \%$, the maximum resolution is $\sim 8 \times 10^{-8}$ RIUs at $\lambda_{\mathrm{o}}=1200 \mathrm{~nm}$ and improves to $\sim \times 10^{-8}$ RIUs at $\lambda_{\mathrm{o}}=1700 \mathrm{~nm}$. The points of maximum resolution for each wavelength are marked by the solid circles. A broadband device can also be realized for Region III by making a trade off between the sensor resolution and the ability to tune the device using the operating wavelength. Fig. 9 shows the sensor's resolution at four different operating points in Region III for $\Delta T_{P}=0.2 \%$ at several wavelengths as a function of the cladding thickness. Fig. 9 also illustrates how $d_{\text {opt }}$ (the apexes of the parabolas) changes with wavelength and operating point. Furthermore, Fig. 9 gives a sense of how sensitive the resolution is to the cladding thickness, $d$.

\section{EXPERIMENTAL SETUP AND RESULTS}

To fabricate a sensor a one-centimetre long stripped section of D-fibre was dipped in acetone for 20 minutes to clean the fibre and then etched in a $10 \%$ hydrofluoric acid solution for 150 to 180 minutes to obtain the desired cladding thickness ${ }^{36,37}$. The cladding thickness after etching can be determined accurately ${ }^{33,34,37}$. We used standard thermo-optic oils to measure the sensor's response. OIL-1 had an index of refraction at $25^{\circ} \mathrm{C}, n_{0}$, and thermo-optic coefficient, $d n / d T$, of 1.4710 and $-3.86 \times 10^{-4} /{ }^{\circ} \mathrm{C}$, respectively, at $\lambda_{0}=1550 \mathrm{~nm}$ and OIL-2 had $n_{0}=1.451$ and $d n / d T=-3.83 \times 10^{-4} /{ }^{\circ} \mathrm{C}$ at $\lambda_{\mathrm{o}}=1550 \mathrm{~nm}$. The thermo-optic coefficients of the oils were valid for a temperature range of $15-35^{\circ} \mathrm{C}$. Outside of this range, the values were not exactly known. Thus to characterize the sensor in Region I, OIL-2 was used and in Region III, OIL-1 was used, keeping their temperatures between $15-35^{\circ} \mathrm{C}$.

The experimental setup is shown in Fig. 10. The D-fibre was threaded through a specially built aluminum plate that had a trench in it to contain the thermo-optic oil. The plate also served as the cold plate for the thermoelectric cooler (TEC) used to control the temperature of the oil. The polarization controller (PC) was used to launch only the $\mathrm{TE}_{01}$ mode in the fibre. To confirm that the $\mathrm{TE}_{01}$ mode was launched correctly, an analyzer was placed at the output to measure the extinction ratio between the two orthogonal modes of the fibre, which we found to be consistently in excess of $27 \mathrm{~dB}$. The ends of the D-fibres were coupled to standard SMF-28 fibres using mechanical splices.

\subsection{Sensor Resolution}

To demonstrate the sensor's high resolution, its response was measured at a single wavelength $\left(\lambda_{0}=1550 \mathrm{~nm}\right)$ using a monochromatic source and a power meter. A sensor with a cladding thickness of $\sim 4.0 \mu \mathrm{m}$ was fabricated and will be referred to as Sensor-1. The power level when the sensor was surrounded by air was used as the reference level to calculate the transmission ratio values. The temperature was changed to change the SRI of the sensor and the measured temperature was used to determine the refractive index of the oil. Fig. 11 shows the measured power transmission ratio of Sensor-1 as a function of the refractive index of OIL-1. The slope in Region III of the measured response was used to determine the sensor's resolution. For $\Delta T_{P}=0.2 \%$, the measured maximum resolution over the range of Region III for which the transmission was measured was $\sim 5 \times 10^{-4}$ at $n_{s} \approx 1.475$ and agrees reasonably well with the calculated resolution of $7 \times 10^{-4}$ shown in Fig. 3. For better temperature control and accurate knowledge of the refractive index of the oil, OIL-2 was used to measure the power transmission ratio of the sensor in Region I, and is shown in Fig. 12. The measured maximum resolution with OIL-2 was determined to be $2 \times 10^{-6}$ at $n_{s} \approx 1.449$ and agrees well with the calculated resolution of $2 \times 10^{-6}$ shown in Fig. 3 .

\subsubsection{Operating Point}

To demonstrate the ability to shift the operating points in either of the two regions, the power transmission ratio was measured at several wavelengths using several monochromatic. At each wavelength, the Cauchy equations and thermooptic coefficients for each oil were used to determine their respective refractive indices. Fig. 13 shows the measured power transmission ratio of Sensor-1 at several wavelengths using OIL-1. To demonstrate the tuneable broadband sensor proposed in Fig. 8, a second sensor, Sensor-2, with a smaller cladding thickness than Sensor-1 was fabricated. Fig. 14 shows the measured power transmission ratio of Sensor-2 using OIL-2. The measured shift of the operating point in Region I shown in Fig. 14 was $~ 0.006$ RIUs, which agrees reasonably well with the calculated shift of 0.007 RIUs shown in Fig. 5, validating the model and the predictions made. The measured maximum resolution for Sensor-2 was $\sim 4.8 \times 10^{-6}$ at $n_{s} \approx 1.4535$ for $\lambda_{\mathrm{o}}=1310 \mathrm{~nm}$ and $\sim 9.8 \times 10^{-7}$ at $n_{s} \approx 1.4481$ for $\lambda_{\mathrm{o}}=1610 \mathrm{~nm}$. 


\section{DISCUSSION}

As we used a thermo-optic oil as the surrounding refractive index, the effects of temperature on the sensor could not be overlooked. The thermo-optic coefficient of silica, which is $-10^{-5} \mathrm{RIU} /{ }^{\circ} \mathrm{C}$, was used to calculate the effect of temperature on the sensor's response. This was done by calculating the refractive indices of the core and cladding at each temperature point and using those values in the model. Fig. 15 shows the power transmission ratio at five different ambient temperatures for a sensor with $L=1 \mathrm{~cm}$ and $d=4.0 \mu \mathrm{m}$ at $\lambda_{0}=1550 \mathrm{~nm}$. The thermo-optic effect in the silica is most pronounced in Region I and has less of an effect in Region III. The shift of Region I due to ambient temperature changes is $\sim 10^{-5} /{ }^{\circ} \mathrm{C}$, indicating that for an accurate measurement of the refractive index, when operated in Region I, the sensor and the surrounding refractive index must be kept at a constant temperature. As a majority of fibre optic sensors are sensitive to temperature changes and require some sort of temperature compensation/control, this is a common requirement.

The measured power transmission ratio curves of the D-fibres shown in the preceding figures illustrate that there is a gradual decrease in $T_{P}$ as $n_{s}$ approaches $n_{\text {eff }}$, as opposed to a sharp decrease predicted by the theoretical model as is shown in the calculated transmission ratio curves. This effect is due to the fact that the thermo-optic oils used are lossy (their transparency decreases with increasing wavelength). Part of the evanescent optical field of the D-fibre propagates in the oil and thus optical power is lost due to absorption in the oil even before the mode becomes leaky, i.e., when $n_{s}<n_{\text {eff. }}$. As $n_{s}$ approaches $n_{\text {eff }}$, the evanescent field penetrates deeper into the oil (the transverse propagation constant decreases) and more of the optical field propagates in the oil, leading to increased absorption loss in the oil. This effect is demonstrated well in Fig. 14 where this gradual decrease gets more pronounced as the wavelength increases.

Since the sensor presented is intensity-based, its response depends on the measurement of the optical transmission, which can fluctuate due to factors such as source temperature changes, detector responsivity changes, link attenuation changes, and those caused by changes in mechanical components such as connectors, mechanical support mounts, and optic $^{38}$. Nevertheless, in an industrial application, compensation techniques commonly used with intensity-based optical fibre sensors can be applied ${ }^{39}$.

\section{CONCLUSIONS}

In this paper, a high resolution refractive index sensor that can be tailored with regard to its operating point and resolution was presented. The power transmission ratio of the sensor depends on the surrounding refractive index when it is greater than the mode effective index. Our sensor had a measured maximum resolution of $2 \times 10^{-6}$ for $\lambda_{0}=1550 \mathrm{~nm}$ at indices close to the effective mode index. We have shown that the best calculated resolution can be $1 \times 10^{-8}$ at $\lambda_{0}=1700 \mathrm{~nm}$. The resolution and the point at which the maximum resolution occurs can be shifted by selecting the correct combination of the cladding thickness and operating wavelength. The maximum resolution point can be shifted by as much as $\sim 0.012$ RIUs in the high resolution region and by $\sim 0.027$ RIUs in the low resolution region with a corresponding wavelength change of $500 \mathrm{~nm}$. The advantages of the sensor are its high resolution, its adjustable operating point, its wide operating range, its manufacturing simplicity, and its implementation using readily available optical sources and simple detection schemes.

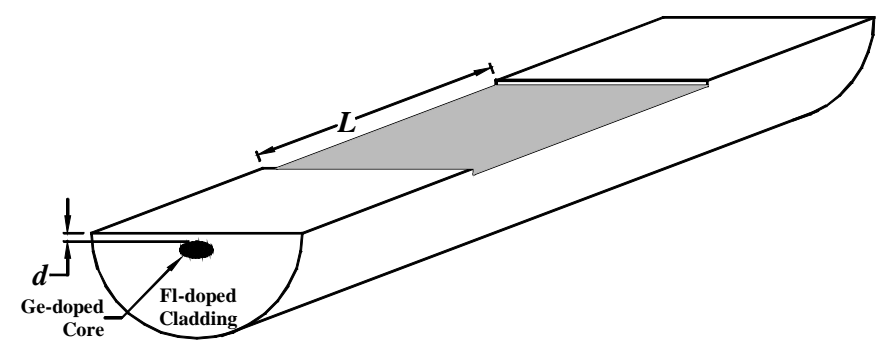

Fig. 1. Conceptual diagram of D-fibre with a section of length $L$ etched to act as sensor head. 


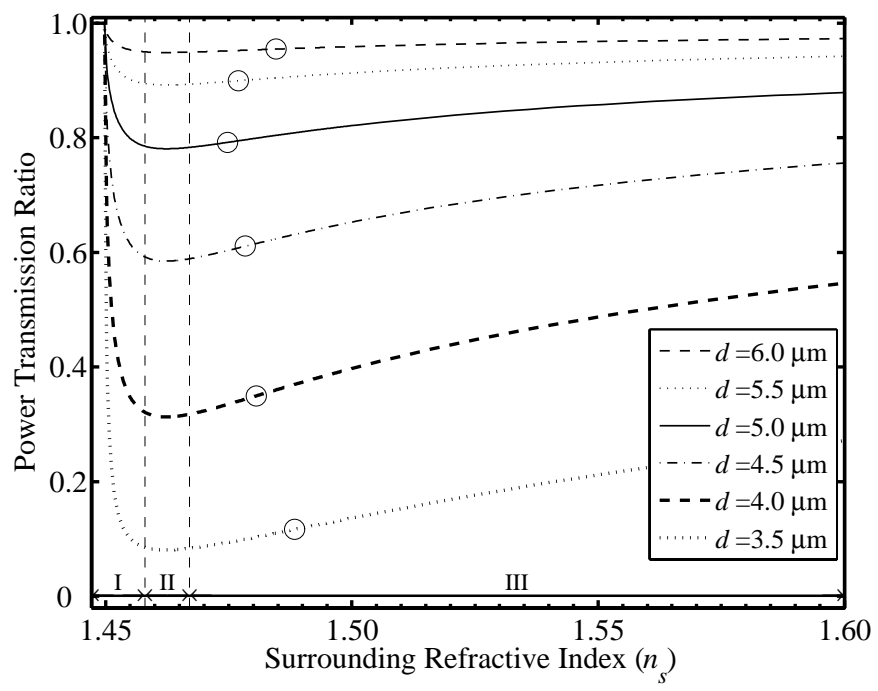

Fig. 2: Calculated power transmission ratio for the equivalent planar waveguide's $\mathrm{TE}_{0}$ mode versus $n_{s}$ for several values of $d$ using $a=2 \mu \mathrm{m}, b=1 \mu \mathrm{m}$ and $L=1 \mathrm{~cm}$ at $\lambda_{\mathrm{o}}=1550 \mathrm{~nm}$. Vertical dotted lines separate Regions I, II, and III. Circles are points where the maximum resolution occurs in Region III.

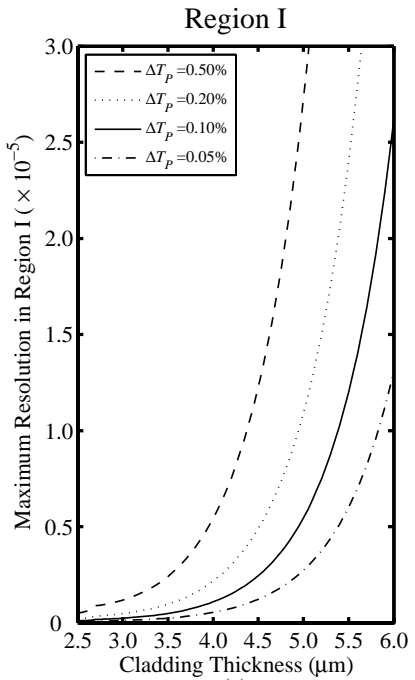

(a)

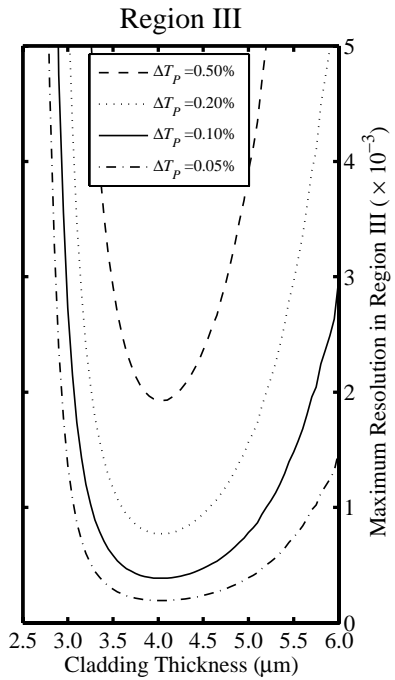

(b)

Fig. 3: Calculated maximum resolution of D-fibre sensor as a function of $d$ for $\Delta T_{P}=0.5 \%, 0.2 \%, 0.1 \%$ and $0.05 \%$ in (a) Region I and (b) Region III. 

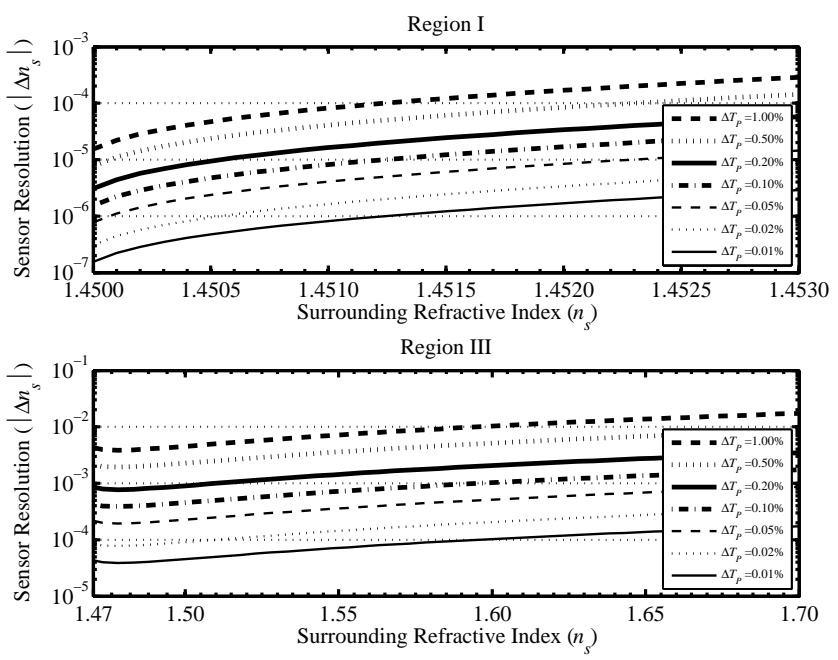

Fig. 4: Calculated resolution of D-fibre sensor with $d=4.0 \mu \mathrm{m}$ as a function of $n_{s}$ for several values of $\Delta T_{P}$ in Region I and Region III.

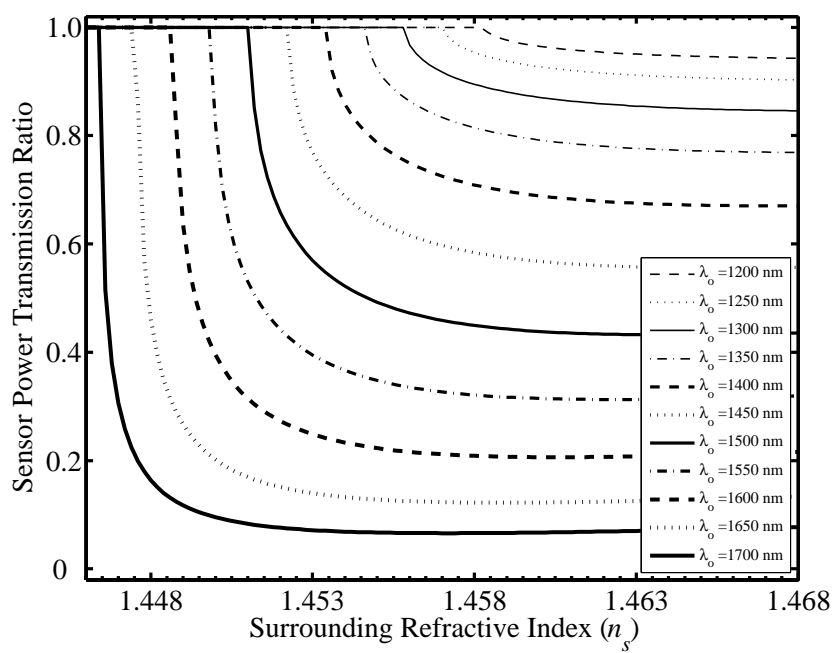

Fig. 5: Calculated power transmission ratio for the $\mathrm{TE}_{0}$ mode of the equivalent planar waveguide in Region I versus $n_{s}$ at several wavelengths using $a=2 \mu \mathrm{m}, b=1 \mu \mathrm{m}, L=1 \mathrm{~cm}$ and $d=4.0 \mu \mathrm{m}$.

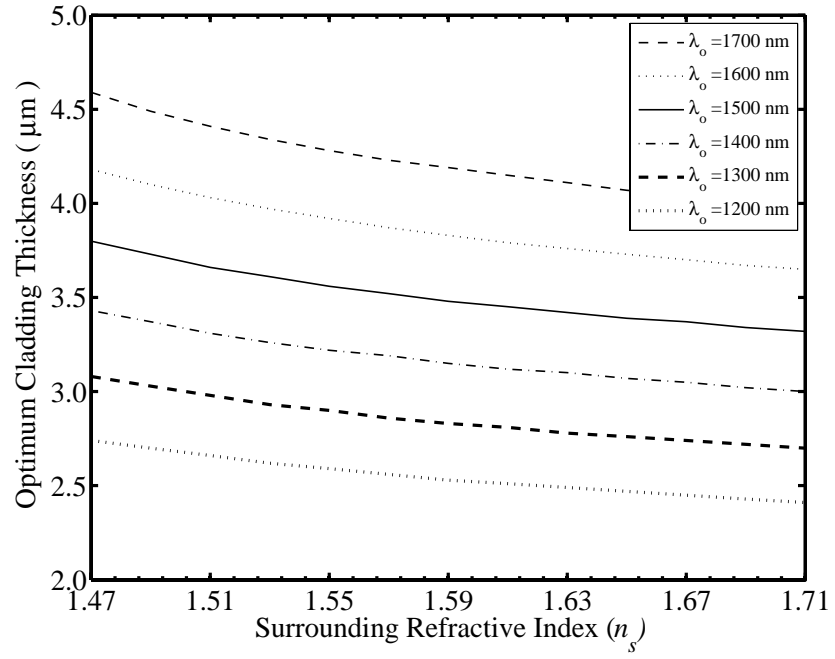

Proc. of SPIE Vol. 7099 709924-7 
Fig. 6: Optimum cladding thickness as a function of $n_{s}$ for several wavelengths.

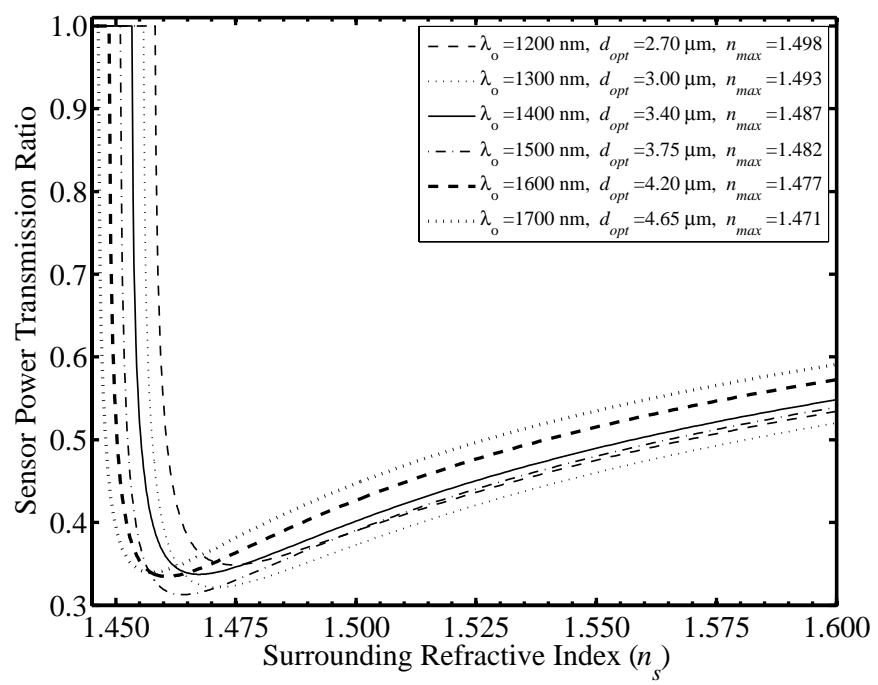

Fig. 7: Calculated power transmission ratio for the equivalent planar waveguide's $\mathrm{TE}_{0}$ mode versus $n_{s}$ for several values of $\lambda_{o}$ using $L=1 \mathrm{~cm}$ and $d_{\text {opt }}$ for each wavelength. The legend shows the point at which the maximum resolution occurs.

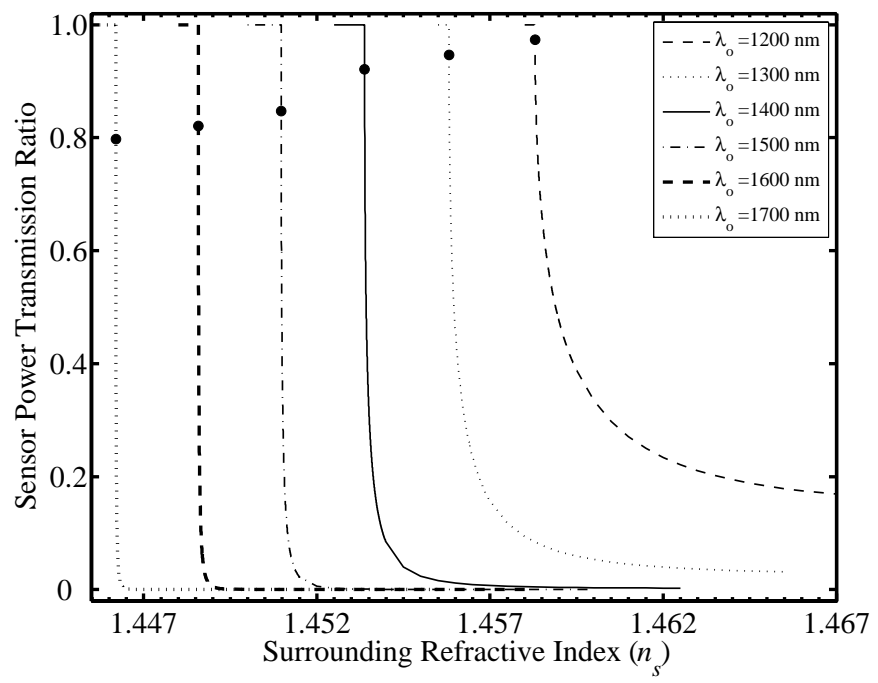

Fig. 8: Power transmission ratio curves for the equivalent planar waveguide's $\mathrm{TE}_{0}$ mode in Region I as a function of $n_{s}$ for $d=2.50 \mu \mathrm{m}$ at several wavelengths. Dark circles mark the points of maximum resolution. 

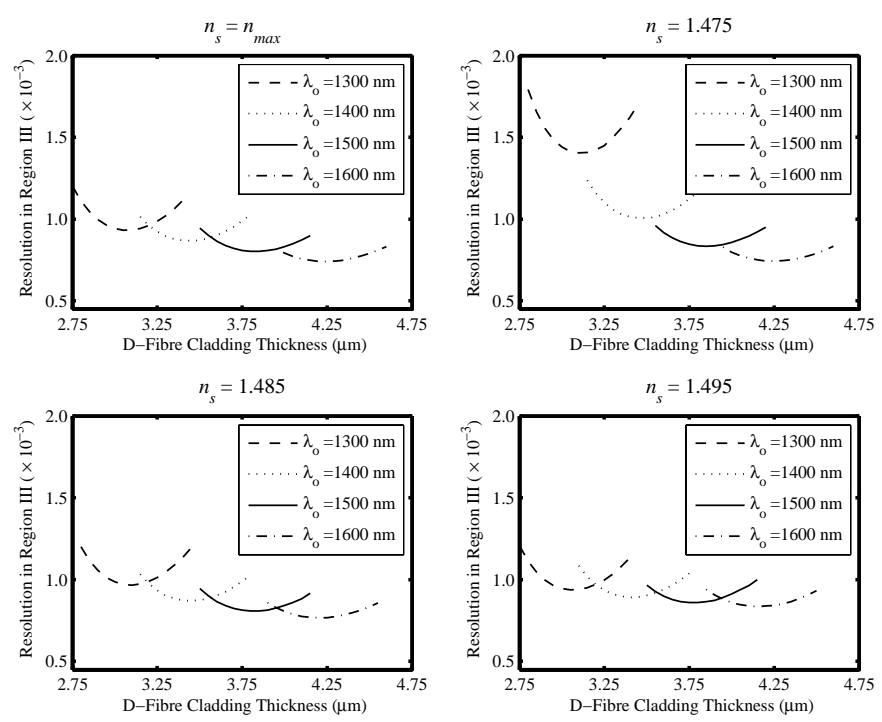

Fig. 9: Sensor resolution in Region III at four different operating points as a function of cladding thickness at several wavelengths using $\Delta T_{P}=0.2 \%$.

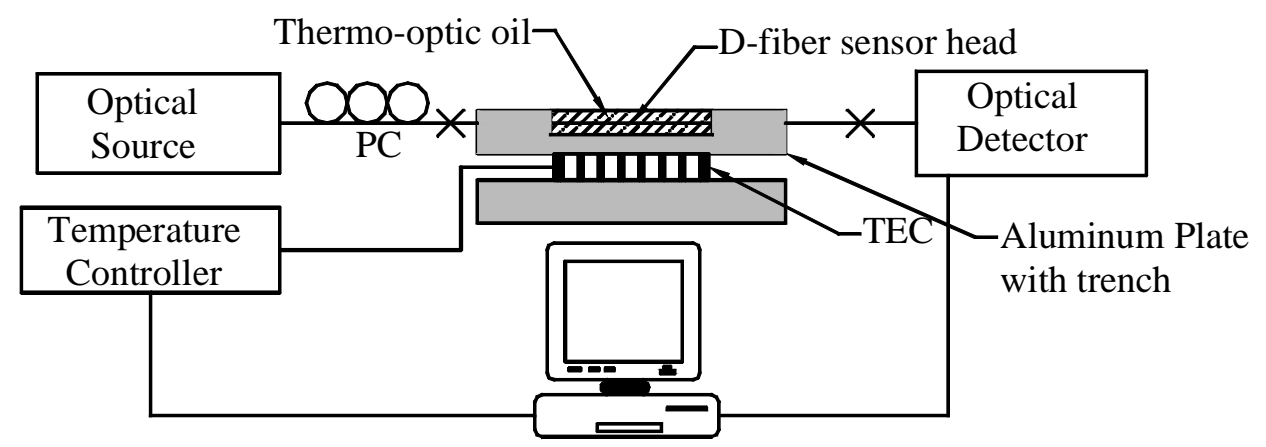

Fig. 10: Experimental setup showing sensor head immersed in thermo-optic oil. TEC- Thermoelectric Cooler; $\times$-Fibre splice; PCPolarization Controller.

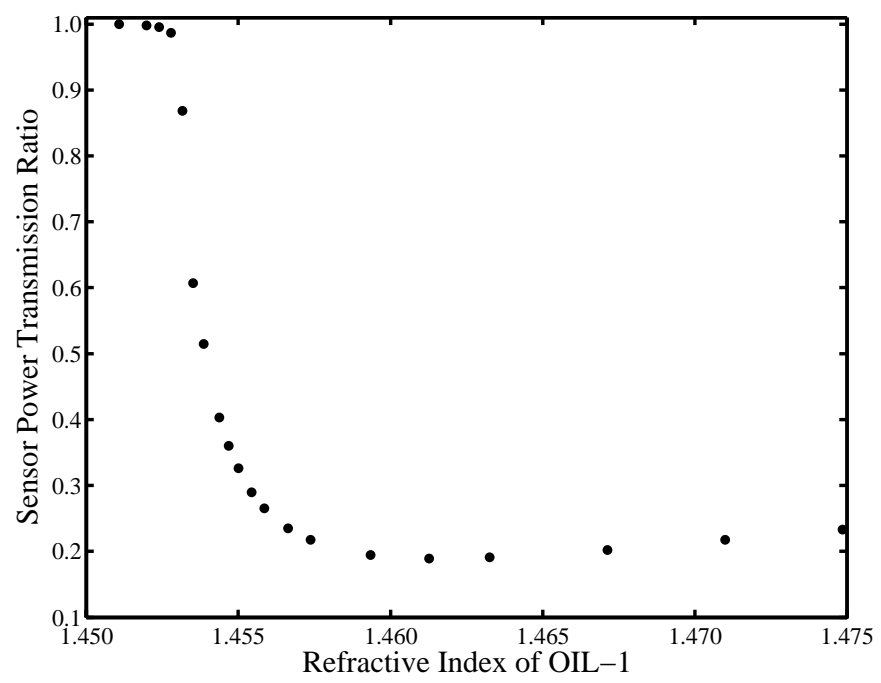

Fig. 11: Measured power transmission ratio of Sensor-1 in all Regions using OIL-1 at $\lambda_{o}=1550 \mathrm{~nm}$. 


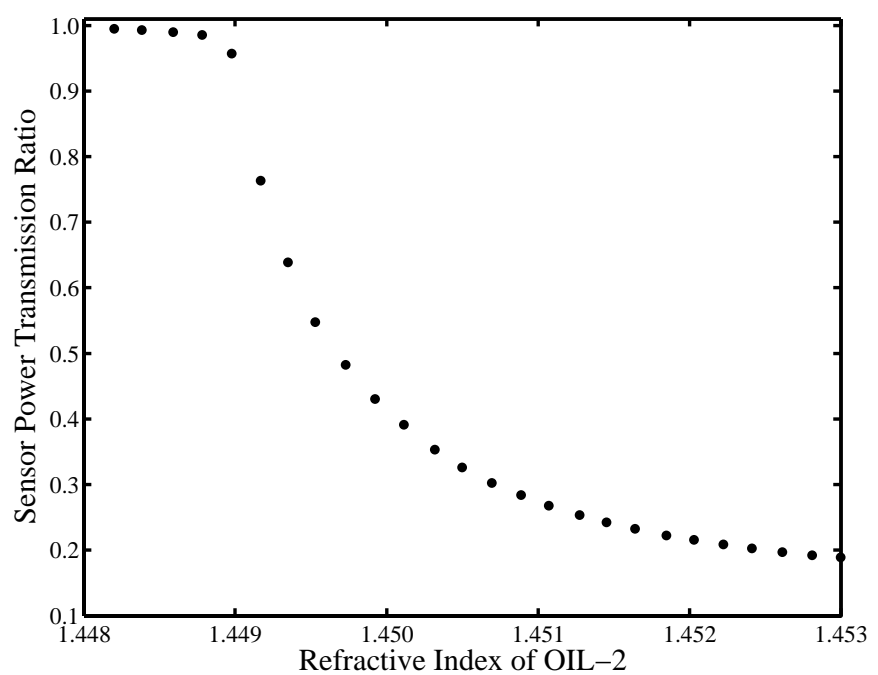

Fig. 12: Measured power transmission ratio of Sensor-1 in Region I using OIL-2 at $\lambda_{\mathrm{o}}=1550 \mathrm{~nm}$.

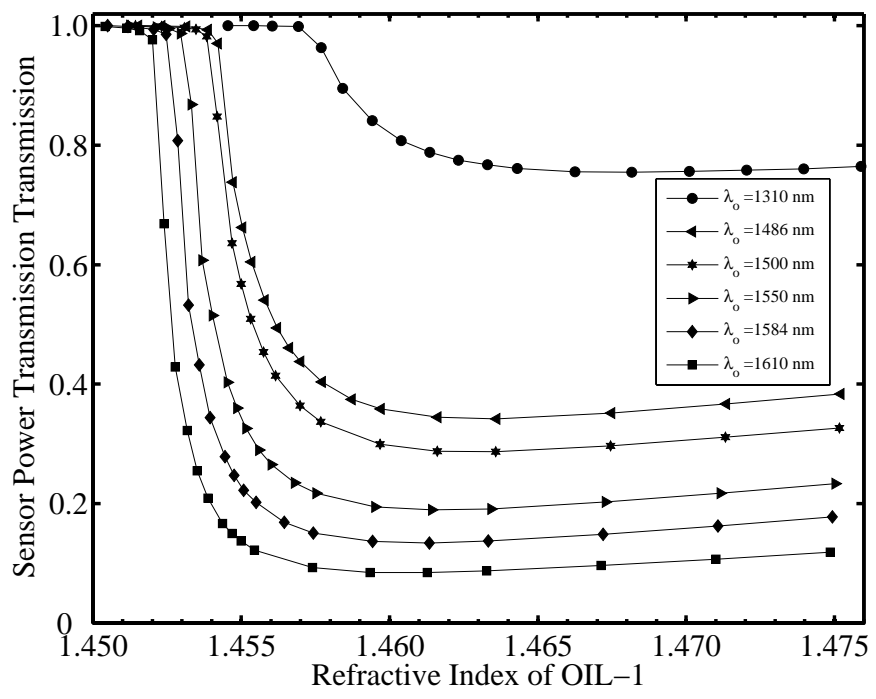

Fig. 13: Measured power transmission ratio of Sensor-1 using OIL-1 at several wavelengths.

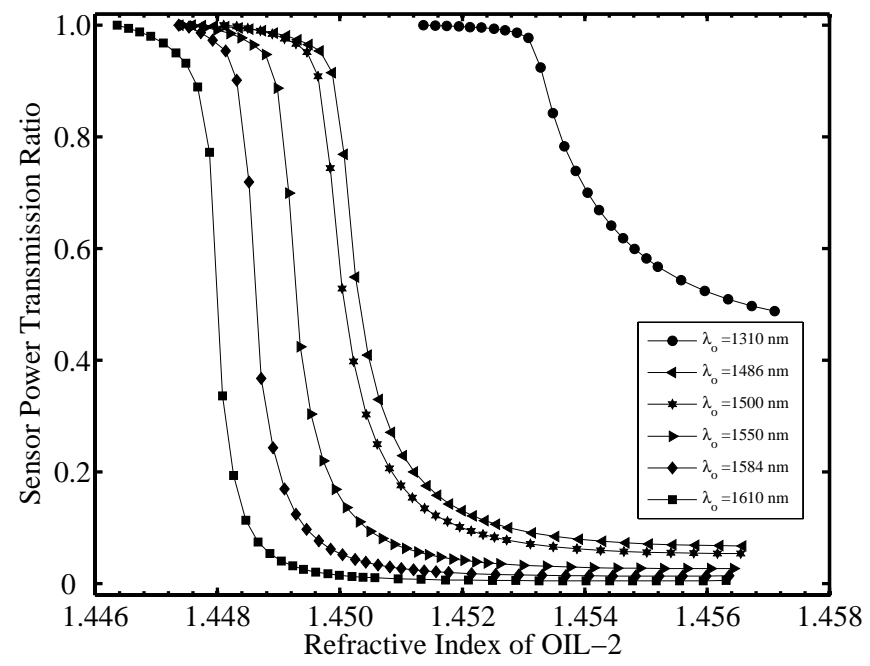

Proc. of SPIE Vol. 7099 709924-10 
Fig. 14: Measured power transmission ratio of Sensor-2 in Region I using OIL-2 at several wavelengths.

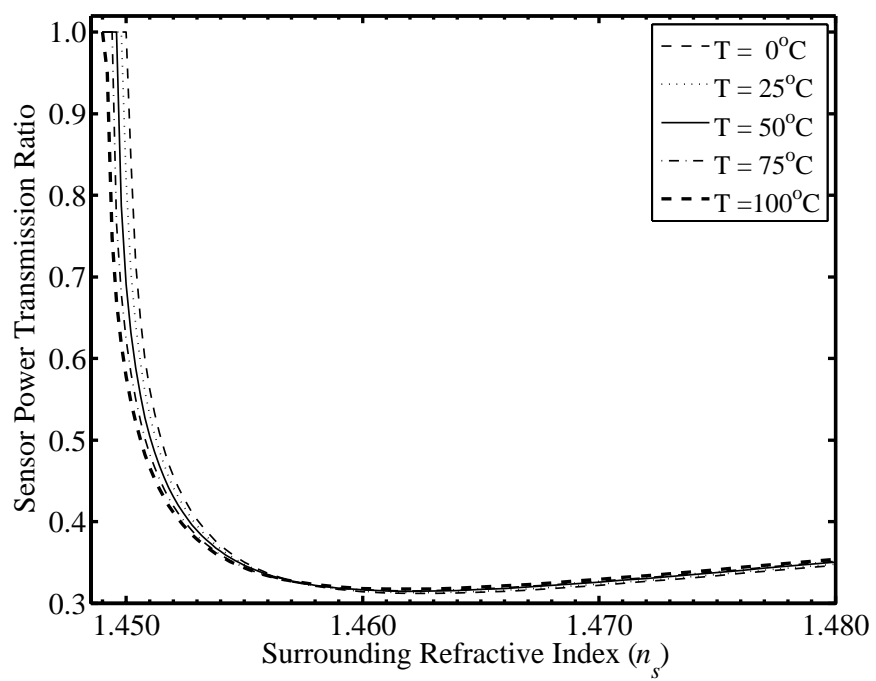

Fig. 15: Calculated power transmission ratio versus surrounding refractive index at various ambient temperatures for a sensor with $L=1 \mathrm{~cm}$ and $d=4.0 \mu \mathrm{m}$ at $\lambda_{\mathrm{o}}=1550 \mathrm{~nm}$.

\section{ACKNOWLEDGMENTS}

This research has been supported in part by the National Sciences and Engineering Research Council of Canada.

\section{REFERENCES}

[1] Jung, Y., Kim, S., Lee, D. S. and Oh, K., "Compact three segmented multimode fiber modal interferometer for high sensitivity refractive-index measurement," Proc. of SPIE 5855, 150-153 (2005).

[2] Villatoro, J., Monzón-Hernández, D., "Low-cost optical fiber refractive-index sensor based on core diameter mismatch ," IEEE JLT 24(3), 1409-1413 (2006).

[3] Chen, X., Zhou, K., Zhang, L., Bennion, I., "Optical chemsensors utilizing long-period fiber gratings UV-inscribed in D-fiber with enhanced sensitivity through cladding etching," IEEE PTL 16(5), 1352-1354 (2004).

[4] Iadicicco, A., Cusano, A., Campopiano, S., Cutolo, A., Giordano, M., "Thinned fiber Bragg gratings as refractive index sensors," IEEE Sensors Journal 5(6), 1288-95 (2005).

[5] Liang, W., Huang, Y., Xu, Y., Lee, R. K., and Yariv, A., "Highly sensitive fiber Bragg grating refractive index sensors," Applied Phys. Let. 86(15), 151122-1-3 (2005).

[6] Chen, N., Yun, B., Cui, Y., "Cladding mode resonances of etch-eroded fiber Bragg grating for ambient refractive index sensing," Applied Phys. Let. 88(13), 133902-1-3 (2006).

[7] Zhou, K., Zhang, L., Chen, X., Bennion, I., "Optic sensors of high refractive-index responsivity and low thermal cross sensitivity that use fiber Bragg gratings of $>80^{\circ}$ tilted structures," Optics Let. 31(9), 1193-5 (2006).

[8] Chan, C-F., Chen, C., Jafari, A., Laronche, A., Thomson, D. J., and Albert, J., "Optical fiber refractometer using narrowband cladding-mode resonance shifts," Applied Optics 46(7), 1142-1149 (2007).

[9] Schroeder, K., Ecke, W., Mueller, R., Willsch, R., Andreev, A., "A fibre Bragg grating refractometer," Meas. Sci. \& Tech. 12(7), 757-64 (2001).

[10] Meltz, G., Hewlett, S. J., Love, J. D., "Fiber grating evanescent-wave sensors," Proc. SPIE 2836, 342-350 (1996).

[11] Huy, M. C. P. et al., "Fibre Bragg grating photowriting in microstructured optical fibres for refractive index measurement," Meas. Sci. \& Tech. 17(5), $992-7$ (2006).

[12] Lee, B. H., Liu, Y., Lee, S. B., Choi, S. S., Jang, J. N., "Displacements of the resonant peaks of a long-period fiber grating induced by a change of ambient refractive index," Optics Let. 22(23), 1769-71 (1997).

[13] Shu, X., Gwandu, B. A. L., Liu, Y., Zhang, L.. Bennion, I., "Sampled fiber Bragg grating for simultaneous refractive-index and temperature measurement," Optics Let. 26(11), 774-6 (2001). 
[14] Ding, J.-F., Zhang, A. P., Shao, L.-Y., Yan, J-H., He, S., "Fiber-taper seeded long-period grating pair as a highly sensitive refractive-index sensor," IEEE PTL 17(6), 1247-9 (2005).

[15] Takeo, T. and Hattori, H., "Optical fiber sensor for measuring refractive index," Jpn. J. of Applied Phys. 1 21(10), 1509-12 (1982).

[16] Villatoro, J., Monzón-Hernández, D., Luna-Moreno, D., "Miniature optical fiber refractometer using cladded multimode tapered fiber tips," Sensors and Actuators B 110(1), 36-40 (2005).

[17] Polynkin, P., Polynkin, A., Peyghambarian, N., Mansuripur, M., "Evanescent field-based optical fiber sensing device for measuring the refractive index of liquids in microfluidic channels," Optics Let. 30(11), 1273-5 (2005).

[18] Falco, L., Spescha, G., Roth, P., Parriaux, O., "Non-ambiguous evanescent-wave fibre refractive index and temperature sensor," Optica Acta 33(12), 1563-70 (1986).

[19] Ribeiro, R. M., Canedo, J. L. P., Werneck, M. M., Kawase, L. R., "An evanescent-coupling plastic optical fibre refractometer and absorption meter based on surface light scattering," Sens. \& Actuators A101(1-2), 69-76 (2002).

[20] Zubia, J., Garitaonaindia, G., Arrue, J., "Passive device based on plastic optical fibers to determine the indices of refraction of liquids," Applied Optics 39(6), 941-6 (2000).

[21] Iadicicco, A., Campopiano, S., Cutolo, A., Giordano, M., Cusano, A., "Refractive index sensor based on microstructured fiber Bragg grating," Photonics Technology Letters 17(6), 1250-2 (2005).

[22] Trudeau et al., J. M., "Combined fibre-optic sensor for colour and refractive index (CI) monitoring," Meas. Sci. \& Tech. 17(5), 1134-9 (2006).

[23] Shao, L.-Y., Zhang, A. P., Liu, W.-S., Fu, H.-Y., He, S., "Optical refractive-index sensor based on dual fiber-Bragg gratings interposed with a multimode-fiber taper," IEEE PTL 19(1), 30-2 (2007).

[24] Homola, J., Yee, S. S., Gauglitz, G., "Surface plasmon resonance sensors: review," Sens. \& Actuators B54 (1-2), 3-15 (1999).

[25] Monzón-Hernández, D., Villatoro, J., "High-resolution refractive index sensing by means of a multiple-peak surface plasmon resonance optical fiber sensor," Sens. \& Actuators B115(1), 227-231 (2006).

[26] Slavík, R., Homola J., and Čtyroký, J., "Single-mode optical fiber surface plasmon resonance sensor," Sens \& Actuators B54(1-2), 74-79 (1999).

[27] Chiu, M-.H, Wang, S-.F. and Chang, R-.S., "D-type fiber biosensor based on surface-plasmon resonance technology and heterodyne interferometry," Optics Let. 30(3), 233-235 (2005).

[28] Marcuse, D., "Investigation of coupling between a fiber and an infinite slab," IEEE JLT 7(1), 122-130 (1989).

[29] Digonnet, M. J. F., Feth, J. R., Stokes, L. F., Shaw, H. J, "Measurement of the core proximity in polished fiber substrates and couplers," Optics Let. 10(9), 463-5 (1985).

[30] Ghatak, A. K., "Leaky modes in optical waveguides," Optical and Quantum Electronics 17(5), 311-21 (1985).

[31] Arnaud, J. A., "Transverse coupling in fiber optics, Part I: Coupling between trapped modes," Bell Sys. Tech. Journal 53(2), 217-24 (1974).

[32] Arnaud, J. A., "Transverse coupling in fiber optics, Part II: Coupling to mode sinks," Bell Sys. Tech. Journal 53(4), 675-96 (1974).

[33] Chandani, S. M., Kulpa, A., Jaeger, N. A. F., "Non-destructive determination of cladding thickness in D-fibers," IEEE PTL 18(9), 1082-1084 (2006).

[34] Chandani, S. M. and Jaeger, N. A. F., "Modelling of elliptical core D-fibers for determination of cladding thickness," Proc. SPIE 6796, (2007).

[35] Dyott, R. B., [Elliptical Fiber Waveguides], Artech House, Norwood, MA, 100-102 (1995).

[36] Chandani, S. M. and Jaeger, N. A. F., "An optical fiber-based liquid level sensor," Optical Engineering 46(11), 114401 (2007).

[37] Chandani, S. M., "Fibre Optic Sensors Based on D-Shaped Elliptical Core Fibres," Ph.D. dissertation, University of British Columbia, Vancouver, B.C., Canada (2007).

[38] Jones, B. E., Medlock, R. S., Spooncer, R. C., "Intensity and Wavelength-Based Sensor and Optical Actuators," Chapter 12 [Optical Fiber Sensors: Systems and Applications 2], Culshaw, B. and Dakin, J., Eds., Artech House, Norwood, 433-434 (1989).

[39] García, A. C., Cuena, J. E., "Transduction Techniques based on Intensity Modulation of Light," Chapter 11 [Handbook of Optical Fibre Sensing Technology], López-Higuera, J. M., Ed., John Wiley \& Sons Ltd., West Sussex, 221-223 (2002). 